\title{
Imagen corporal (IC) y estrategias de afrontamiento (EA): Análisis de las características médico quirúrgicas en mujeres con diagnóstico de cáncer de mama
}

\author{
Body image (BI) and coping strategies (CS): Analysis of medical and surgical \\ characteristics in women diagnosed with breast cancer
}

\author{
César Núñez \\ Universidad de Medellín, Colombia \\ Ana Navarro \\ Sebastián Cortés \\ Universidad Autónoma de Bucaramanga y Corporación Universitaria \\ Alexander von Humboldt, Colombia \\ Jessica López \\ Universidad Santiago de Cali, Colombia \\ Haney Aguirre-Loaiza \\ Universidad Católica de Pereira, Colombia \\ Catalina Trujillo \\ Universidad del Valle, Colombia
}

Recepción (10 de octubre de 2017) Aceptado (16 de mayo de 2018)

Correspondencia: Instituto de Psicología. Universidad del Valle, Ciudad Universitaria Meléndez. Calle 13 No 100-00. Edificio 388 , Oficina 4040. Santiago de Cali, Colombia

César Núñez: Investigador Programa de Psicología. Universidad de Medellín. Medellín, Colombia. Email: cnunez@udem.edu.co

Ana Navarro: Programa de Psicología extensión Armenia. Universidad Autónoma de Bucaramanga. Médico Hospital San Juan de Dios. Armenia,

Colombia. Email: anavarro462@ cue.edu.co

Sebastián Cortés: Universidad Autónoma de Bucaramanga, extensión Armenia. Armenia, Colombia. Email: sscortes7@gmail.com

Jessica López: Mg. Psicología Cognitiva. Universidad de Buenos Aires. Docente Universidad Santiago de Cali. Cali, Colombia. Email: jessica.

lopezpelaez@gmail.com

Haney Aguirre-Loaiza: Mg. en Psicología. Universidad del Valle. Docente Universidad Católica de Pereira, Colombia. Email:

haney.aguirre@ucp.edu.co

Catalina Trujillo: Mg(c) en Psicología. Universidad del Valle. Cali, Colombia. Email: catalinatrujillo.llano@gmail.com

Agradecimientos y financiación:

A las mujeres participantes, Oncólogos de Occidente-sede Armenia, la Fundación Lazo Rosa, estudiantes de Psicología de la Corporación

Universitaria Alexander Von Humboldt, participantes en la recolección de la información.

Proyecto financiado por la Corporación Universitaria Alexander von Humboldt, Universidad de Medellín y Universidad Santiago de Cali. 


\title{
Resumen
}

A partir de un diseño No-experimental transversal, se buscó identificar la posible relación entre la IC y las EA, y sus diferencias según variables médico quirúrgicas (intervención quirúrgica, tiempo de diagnóstico y estadio del cáncer). Participaron treinta y siete mujeres diagnosticadas con cáncer de mama de todos los estadios. Se usó el Cuestionario de Afrontamiento al Estrés para Pacientes Oncológicos (CAEPO), la Escala de Imagen Corporal (BIS) y un cuestionario Ad-hoc. No se encontró relación entre la IC y las EA, y éstas no varían según la intervención quirúrgica (mastectomía radical y cirugía conservadora), el tiempo de diagnóstico en años (menos de 1 , entre 1 y 3 , entre 3 y 5 , y más de 5), o el estadio ( 0 o in situ, I, II, III y IV); sin embargo, las pacientes que no recibieron intervención quirúrgica evidenciaron mayor deterioro de su IC que aquellas que sí lo hicieron.

Palabras clave: Imagen corporal, Cáncer de seno, Estrategias de afrontamiento, Oncología (DeCS).

\begin{abstract}
From a non-experimental transverse design, we sought to identify the possible relationship between BI and $\mathrm{CS}$, and their differences according to surgical-medical variables (surgical intervention, time of diagnosis and stage of cancer). Thirty-seven women diagnosed with breast cancer from all stages participated. The Stress Coping Questionnaire for Oncological Patients (CAEPO), the Body Image Scale (BIS) and an Ad-hoc questionnaire were used. No relationship was found between BI and CS, and these did not vary according to the surgical procedure (radical mastectomy and conservative surgery), the time of diagnosis in years (less than 1, between 1 and 3, between 3 and 5, and more of 5), or the stage ( 0 or in situ, I, II, III and IV); however, the patients who did not receive surgical intervention showed greater deterioration of their BI than those who did.

Keywords: Body image, Breast Neoplasms, Coping behavior, Oncology (DeCS).
\end{abstract}




\section{Introducción}

El cáncer es un conjunto de enfermedades caracterizado por la propagación y crecimiento descontrolado de células anormales, las cuales generan masas o tumores y reciben su nombre según la parte del cuerpo o tejido en que se originan (Weinberg, 2013). El cáncer de seno es la primera causa de mortalidad en mujeres, seguida por el de colon, recto, pulmón, cuello uterino y estómago (WHO, 2015). En Colombia, esta enfermedad se perfila como un problema de salud pública debido a que se reportan 8.686 casos nuevos y causa la muerte de 2.649 mujeres anualmente (Ministerio de Salud y Protección Social, 2014).

La literatura coincide en que el diagnóstico, tratamiento y recuperación del cáncer constituyen un evento estresante (Font \& Cardoso, 2009; Mcdonough, Sabiston \& Wrosch, 2014), ya que los acontecimientos que lo configuran (cirugías, terapias, hospitalizaciones, dolor, malestar, etc) amenazan la integridad física y/o psicológica del paciente y sus familiares (González, 2008a), afectando su calidad de vida (Barnaś, Skręt-Magierło, Skręt \& Bidziński, 2012; Hopwood, Haviland, Mills, Sumo \& Bliss, 2007; Mera \& Ortiz, 2012) y presentando incluso posibles comorbilidades con el estrés, ansiedad y depresión (Brown \& Kroenke, 2009; Burgess et al., 2005).

\section{Imagen corporal y cáncer de seno}

La imagen corporal (IC) es entendida como la representación mental del propio cuerpo y la actitud sobre la apariencia física, integridad, sexualidad y el estado de salud (Fobair et al., 2006). Esta dimensión suele alterarse en el tratamiento del cáncer de seno debido a los cambios temporales y permanentes, como: caída del cabello, cambios de peso, cicatrices, pérdida parcial o total de uno o los dos senos, entre otros (Hopwood et al., 2007; Rincón, Pérez, Borda \& Martín, 2012; White, 2000). Paralelamente, tales cambios parecen estar asociados con alteraciones sexuales, afectivas y autoestimaen el ciclo de los tratamientos (Ahmed, Marchand, Williams, Coscarelli \& Ganz, 2016).

De acuerdo a lo anterior, la satisfacción con la IC parece diferir según el tipo de intervención quirúrgica recibida. Algunos estudios sostienen que la mastectomía es la opción terapéutica que genera mayor ansiedad y deterioro en la IC (Olivares, Martin, Roman \& Moreno, 2010), y es predictor de vergüenza e insatisfacción con la apariencia física en los seis meses posteriores a la misma (Moreira \& Canavarro, 2010); mostrando mayor afectación en los componentes cognitivo, afectivo y conductual que la cirugía conservadora (Fobair et al., 2006; Olivares et al., 2010; Shoma et al., 2009). Por otro lado, técnicas como la reconstrucción mamaria facilitan la restitución de la IC, el bienestar psicológico, la satisfacción estética y la autoestima (Marin \& Sánchez, 2010; Rincón et al., 2012).

La literatura indica que la IC es cambiante en el tiempo posterior al diagnóstico de la enfermedad oncológica. Al respecto, se han evidenciado problemas sexuales y de IC en pacientes jóvenes en los primeros meses posteriores a éste (Fobair et al., 2006), y se ha reportado que en mujeres mastectomizadas, un mayor tiempo de diagnóstico se relaciona con una mejor IC y disfrute sexual, menor preocupación por el futuro y reducción de síntomas médicos (Rincón et al., 2012). Sin embargo, otros estudios contradicen lo anterior al concluir que las pacientes con mayor tiempo de diagnóstico tienen una IC más deteriorada que aquellas con diagnóstico menor a un año (Hartl et al., 2003), y que la vergüenza (dimensión de la IC) aumenta en los seis meses posteriores a la terminación del tratamiento (Moreira \& Canavarro, 2010). En ambos casos, los cambios subjetivos percibidos afectan la condición de bienestar emocional de las pacientes (Brunet, Sabiston \& Burke, 2013). Ello revela inconsistencias en el cuerpo del conocimiento y resalta la necesidad de atender estos cuestionamientos con nuevos estudios.

En cuanto al estadio oncológico, se ha descrito que a mayor avance de este, mayor deterioro de la IC (Hartl et al., 2003). Sin embargo, el instrumento usado en este estudio representa sólo dos ítems de la IC a un cuestionario de calidad de vida, por lo que sus resultados deben ser asumidos con cautela, y en este caso preferimos estudiar el constructo de la IC con un instrumento específico. Al respecto, la literatura es escasa al considerar variables mediadoras como el estadio y la evolución posterior al diagnóstico.

\section{Estrategias de afrontamiento y cáncer de seno}

El cáncer como un evento estresante, desde el diagnóstico hasta la recuperación, implica la utilización de recursos psicológicos para adaptarse. Una de las variables que mejor predice la calidad de vida y el ajuste psicológico de las pacientes con cáncer de seno son las estrategias de afrontamiento (EA) (Mera \& Ortiz, 2012). Las EA se definen como los esfuerzos cognitivos y conductuales para manejar el estrés y las tensiones causadas por situaciones aversivas y se clasifican en: estrategias de resolución de problemas, dirigidas a modificar o alterar el problema que causa el malestar; y estrategias de regulación emocional, que modulan la repuesta emocional ante éste (Folkman, Lazarus, Gruen \& Delongis, 1986). Así, en la medida en que las EA que utilice un sujeto sean efectivas para solucionar el problema o reducir el malestar, podrán contribuir a una mejor adaptación del individuo a la situación adversa; de lo contrario, la impiden o la interfieren (González, 2008b).

Aunque algunos estudios (De-Haro et al., 2014; Zucca, Boyes, Lecathelinais \& Girgis, 2010) han analizado la 
variabilidad de las EA en función al tipo de tratamiento recibido; específicamente la asociación entre EA y tipo de intervención quirúrgica, se ha estudiado poco. Así, se ha reportado que en pacientes con diagnóstico reciente, a mayor número de tratamientos recibidos, menor capacidad de resolución y evitación de problemas (De-Haro et al., 2014). Asimismo, en mujeres sobrevivientes, después de cinco o seis años de diagnóstico la quimioterapia fue la variable que mejor predijo el uso de todas las EA evaluadas (impotencia, preocupación ansiosa, espíritu luchador, evitación cognitiva y fatalismo) (Zucca et al., 2010).

Otra posible fuente de variabilidad de la EA parece ser el tiempo después del diagnóstico. En un estudio longitudinal con mujeres sobrevivientes a seis años de diagnóstico, se encontró que el afrontamiento en respuesta al cáncer cambió en el tiempo, aunque ello no se relacionó con el riesgo de recaída (Geyer, Koch-Giesselmann \& Noeres, 2015). Además, se ha reportado que las sobrevivientes continúan utilizando EA específicas del cáncer, tales como fatalismo y espíritu luchador, cinco o seis años después de éste, aunque en menor medida que las mujeres con diagnóstico reciente (Zucca et al., 2010); lo cual coincide con resultados que evidencian que el uso de EA decrece con el paso del tiempo (Nosarti, Roberts, Crayford, McKenzie \& David, 2002). Lo anterior podría relacionarse con que las pacientes presentan mayores niveles de ansiedad un mes y medio posterior al diagnóstico (fase en espera del tratamiento), decreciendo en los seis y doce meses siguientes (Silva, Crespo \& Canavarro, 2012). Por otro lado, se ha propuesto que tal decrecimiento es desigual, demostrando que el uso de algunas EA disminuyó, mientras el de otras incrementó (Danhauer, Crawford, Farmer \& Avis, 2009).

En esta línea, la revisión de la literatura no ha mostrado suficiente evidencia en la posible asociación entre la IC y EA. Cabe acotar que aunque las escasas iniciativas no describen una relación entre éstas variables, se ha señalado que las mujeres con cáncer de seno con mejor IC presentan mayor autoeficacia en el afrontamiento (habilidad percibida para afrontar) que aquellas con deterioro de ésta. Así, las mujeres diagnosticadas que se sienten mejor con sus cuerpos, confían más en su habilidad para seguir adelante pese a las consecuencias, afrontar la pérdida de cabello y la disminución de energía, mantenerse esperanzadas y positivas, preguntar a los profesionales por cuestiones médicas, escapar de los aspectos negativos, expresar emociones negativas y buscar apoyo emocional (Pikler \& Winterowd, 2003).

De esta manera, es posible pensar que las mujeres con mejor IC difieren en el uso de EA, lo cual puede estar determinado por las demandas y requerimientos a las que deben se deben enfrentar, varían en su naturaleza e in- tensidad (situación clínica distinta). Además, es posible estimar que la IC y las EA también varían en función al tipo de intervención quirúrgica, tiempo de diagnóstico y estadio del cáncer. Aunque existen estudios que analizan las variables propuestas, los resultados son inconsistentes e inconclusos. Estudiar la relación entre características propias de la enfermedad con variables psicológicas específicas como la IC y la EA permitirá fundamentar el cuerpo del conocimiento y contribuir al mejoramiento y creación de técnicas de intervención integrales y eficaces. El presente trabajo hace parte de una investigación con varias fases metodológicas que está recolectando muestras en otras ciudades de Colombia y apunta a tales propósitos (AguirreLoaiza, Núñez, Navarro, \& Cortés, 2017); por lo tanto, los resultados aquí presentados son parte de una línea de investigación en el tema.

Según lo anterior, los objetivos propuestos fueron: (a) estudiar las posibles diferencias en la IC y las EA en función de variables médico quirúrgicas (intervención quirúrgica, el tiempo de diagnóstico y el estadio del cáncer) en las pacientes y (b) analizar la posible relación entre la IC y las EA en mujeres diagnosticadas con cáncer de seno.

\section{Método}

\section{Participantes}

A partir de un estudio No-Experimental transversal, participaron treinta y siete mujeres, con un rango de edad entre $\operatorname{los} 30$ y 87 años $\left(M_{e d a d}=55.7 ; D E=13.1\right)$, asistidas en Oncólogos de Occidente S.A. (Armenia, Quindío). El tamaño reducido de la muestra se debió al acceso restringido a la base de datos de las mujeres asistentes a la consulta médica y a que la participación estuvo supeditada a su voluntad. Las características sociodemográficas y médicas son descritas en la tabla 1. 
Tabla 1. Características sociodemográficas y médicas

\begin{tabular}{lll}
\hline Estrato Socioeconómico & $\mathrm{N}$ & $\%$ \\
\hline Uno & 9 & 23.1 \\
Dos & 18 & 46.2 \\
Tres & 6 & 15.4 \\
Cuatro & 4 & 10.3 \\
Cinco & 2 & 5.1 \\
Seis & 0 & 0.0 \\
\hline Estado civíl & & \\
\hline Soltero & 8 & 20.5 \\
Casado & 8 & 20.5 \\
Unión libre & 9 & 23.1 \\
Separada & 7 & 17.9 \\
Viudo & 7 & 17.9 \\
\hline Escolaridad & & \\
\hline Primaria & 13 & 33.3 \\
Secundaria & 19 & 48.7 \\
Técnico & 4 & 10.3 \\
Tecnológico & 3 & 7.7 \\
Pregrado & 0 & 0.0 \\
\hline
\end{tabular}

\begin{tabular}{lll}
\hline Tiempo de Diagnóstico & $\mathrm{N}$ & $\%$ \\
\hline Menos de un año & 10 & 25,6 \\
Entre uno y tres años & 12 & 30,8 \\
Entre tres y cinco años & 6 & 15,4 \\
Más de cinco años & 11 & 28,2 \\
\hline Estadio o Etapa & & \\
\hline Estadio 0 & 4 & 10,3 \\
Estadio I & 11 & 28,2 \\
Estadio II & 10 & 25,6 \\
Estadio III & 10 & 25,6 \\
Estadio IV & 4 & 10,3 \\
\hline Intervención Quirúrgica & & \\
\hline Mastectomía radical & 12 & 30,8 \\
Cirugía conservadora & 16 & 41,0 \\
Ninguna & 11 & 28,2 \\
\hline
\end{tabular}

\section{Técnicas e instrumentos}

\section{Imagen Corporal}

La Escala de Imagen Corporal (BIS, por sus siglas en inglés) es un instrumento que evalúa la imagen corporal en pacientes oncológicos (Hopwood, Fletcher, Lee \& Al Ghazal, 2001). Consta de diez ítems que tratan dimensiones comportamentales, afectivas y cognitivas, con una puntuación tipo Likert (1 nunca, y 4 mucho). Su puntaje natural tiene un rango entre $0-30$, de manera que a mayor puntaje, mayor deterioro de la IC (Juárez \& Landero, 2011). Los estudios sobre las propiedades psicométricas del BIS evidencian puntuaciones satisfactorias de consistencia interna $(\alpha=93)$ y validez divergente y convergente (Moreira, Silva, Marques \& Canavarro, 2010). Este estudio estimó un valor de fiabilidad $\alpha=93$, calculado a través del método de consistencia interna.

\section{Estrategias de Afrontamiento}

El Cuestionario de Afrontamiento al Estrés para Pacientes Oncológicos (CAEPO) está compuesto por 40 ítems divididos en sietes escalas y éstas, a su vez, en dos partes (EA positivas y negativas), de 20 ítems cada una. Sus puntajes son convertidos en decatipos.

Estrategias de afrontamiento positivas: Exploran la presencia de conductas y pensamientos positivos y activos, y posibles soluciones para mejorar la situación. Éstas son: Enfrentamiento y Lucha Activa (E1A, 9 ítems); Autocontrol y Control Emocional (ACE, 7 ítems) y Búsqueda de Apoyo Social (BAS, 4 ítems). Los puntajes altos indican la presencia de estrategias de afrontamiento positivas y activas.

Estrategias de afrontamiento negativas: Exploran la presencia de conductas y pensamientos poco o nada adaptativos. Éstas son: Ansiedad y Preocupación Ansiosa (APA, 6 ítems), Pasividad, Resignación Pasiva (PrP, 5 ítems), Huida y Distanciamiento (HD, 6 ítems) y Negación (3 ítems). Los puntajes altos indican situaciones excesivamente amenazantes e incapacidad para afrontarlas (González, 2004, 2008a).

Las puntuaciones del CAEPO han evidenciado validez de constructo a través del análisis factorial exploratorio y validez de criterio, empleando la entrevista clínica como criterio externo. Asimismo, los coeficientes de confiabilidad de alfa de cronbach en las respectivas escalas oscilan entre .78 y .91 (González, 2004). 


\section{Procedimiento y consideraciones éticas}

La información fue recolectada por estudiantes de último año de Psicología y psicólogos profesionales en el centro médico de Oncólogos de Occidentes, sede Armenia, Quindío. Mediante la firma del consentimiento informado se garantizó la claridad sobre los propósitos del estudio, confidencialidad, privacidad y autonomía de retiro. La aplicación del protocolo de evaluación se realizó momentos previos a la consulta con el médico especialista. Se contó con el aval del comité de bioética de la Corporación Universitaria Alexander Von Humboldt y, asimismo, se consideraron las disposiciones de la Resolución 8430 (Ministerio de Salud República de Colombia, 1993), los lineamientos de la Ley 1090 y las regulaciones del Código Deontológico y Bioético del ejercicio de la Psicología en Colombia (Colegio Colombiano de Psicólogos, 2012).

\section{Análisis de datos}

Los datos fueron analizados a través de estadística descriptiva (medidas de tendencia central y de dispersión) e inferencial mediante el SPSS v.20. Posterior al cumplimiento de los supuestos de normalidad (Shapiro-Wilk, $n<50$ ) y homocedasticidad (prueba de Levenne), se utilizaron los estadísticos de correlación $r$ de Pearson y la comparación de medias independientes $t$ de student y AnOVA de un solo factor. Se asumió un nivel de significancia menor a .05 $(p<.05)$. Los resultados de los instrumentos fueron analizados a partir de los puntajes directos.

\section{Resultados}

\section{Imagen corporal y estrategias de afrontamiento según la intervención quirúrgica, el tiempo de diagnóstico y estadio}

Los datos de IC y EA según la intervención quirúrgica, tiempo de diagnóstico y estadio del cáncer describieron un comportamiento homogéneo (tablas 2, 3 y 4). Específicamente, las mujeres que no han recibido ninguna intervención quirúrgica presentaron mayor afectación de la IC en comparación de aquellas que sí la han recibido $F(2,34)=$ $3.54, p=.04$ (Tabla 4).

Tabla 2. Anova de imagen corporal y Estrategias de afrontamiento según la intervención quirúrgica

\begin{tabular}{|c|c|c|c|c|c|c|c|c|}
\hline \multicolumn{9}{|c|}{ INTERVENCIÓN QUIRÚRGICA } \\
\hline \multirow[t]{2}{*}{ Variable } & \multicolumn{2}{|c|}{$\begin{array}{c}\text { Mastectomía } \\
\text { Radical } \\
(n=11)\end{array}$} & \multicolumn{2}{|c|}{$\begin{array}{c}\text { Cirugía } \\
\text { Conservadora } \\
(n=14)\end{array}$} & \multicolumn{2}{|c|}{$\begin{array}{l}\text { Ninguna } \\
(n=12)\end{array}$} & \multirow[t]{2}{*}{$\begin{array}{c}F \\
(g l .2)\end{array}$} & \multirow[t]{2}{*}{$p$} \\
\hline & $M$ & $D E$ & $M$ & $D E$ & $M$ & $D E$ & & \\
\hline \multicolumn{9}{|c|}{ Imagen corporal } \\
\hline BIS & 15.6 & 3.8 & 17.9 & 4.9 & 22.3 & 8.8 & 3.54 & $.040 *$ \\
\hline \multicolumn{9}{|c|}{ estrategIas de afrontamIento } \\
\hline ELA & 23.5 & 3.3 & 23.7 & 3.4 & 23.4 & 3.8 & 0.36 & .965 \\
\hline ACE & 17.2 & 2.7 & 16.5 & 3.2 & 16.5 & 3.5 & 0.20 & .819 \\
\hline BAS & 7.4 & 2.4 & 8.2 & 2.5 & 7.9 & 3.3 & 0.26 & .765 \\
\hline $\operatorname{Sub}(+)$ & 48.2 & 5.6 & 48.5 & 7.5 & 50.5 & 5.8 & 0.02 & .977 \\
\hline APA & 6.0 & 3.8 & 3.43 & 3.6 & 5.8 & 4.7 & 1.62 & .212 \\
\hline PRP & 8.73 & 3.9 & 9.50 & 2.8 & 8.4 & 4.5 & .282 & .756 \\
\hline HD & 9.2 & 3.3 & 8.57 & 3.0 & 9.0 & 3.7 & .137 & .872 \\
\hline $\mathrm{N}$ & 3.4 & 2.3 & 4.14 & 2.7 & 3.7 & 2.3 & .234 & .793 \\
\hline $\operatorname{Sub}(-)$ & 27.4 & 10.2 & 25.6 & 6.5 & 25.5 & 10.9 & 0.14 & .866 \\
\hline
\end{tabular}

Nota: $\mathrm{ELA}=$ enfrentamiento y lucha activa, ACE= autocontrol y control emocional, BAS= búsqueda de apoyo social, Sub(+)= subtotal de las escalas de estrategias de afrontamiento positivas, $\mathrm{APA}=$ ansiedad y preocupación ansiosa, $\mathrm{PRP}=$ pasividad y resignación pasiva, $\mathrm{HD}=$ huida $\mathrm{y}$ distanciamiento, $\mathrm{N}=$ negación, $\mathrm{Sub}(-)=$ subtotal de las escalas de estrategias de afrontamiento negativas, BIS= Body Image Scale $($ Escala de imagen corporal). * $\mathrm{p}<.05$ 
Tabla 3. Anova de imagen corporal y estrategias de afrontamiento según el tiempo de diagnóstico

\begin{tabular}{|c|c|c|c|c|c|c|c|c|c|c|}
\hline \multicolumn{11}{|c|}{ TIEMPO DE DIAGNÓSTICO (EN AÑOS) } \\
\hline \multirow[t]{2}{*}{ Variable } & \multicolumn{2}{|c|}{$\begin{array}{c}<1 \\
(n=8)\end{array}$} & \multicolumn{2}{|c|}{$\begin{array}{c}\text { Entre 1-3 } \\
(n=12)\end{array}$} & \multicolumn{2}{|c|}{$\begin{array}{c}\text { Entre 3-5 } \\
(n=5)\end{array}$} & \multicolumn{2}{|c|}{$\begin{array}{c}>5 \\
(n=8)\end{array}$} & \multirow{2}{*}{$\begin{array}{c}F \\
(\mathrm{gl} .3)\end{array}$} & \multirow{2}{*}{$p$} \\
\hline & $M$ & $D E$ & $M$ & $D E$ & $M$ & $D E$ & $M$ & $D E$ & & \\
\hline \multicolumn{11}{|c|}{ Imagen corporal } \\
\hline BIS & 20.7 & 7.4 & 17.1 & 6.8 & 18.8 & 3.1 & 17.0 & 6.1 & 0.62 & .608 \\
\hline \multicolumn{11}{|c|}{ estrategIas de afrontamIento } \\
\hline ELA & 24.5 & 1.8 & 23.7 & 3.8 & 23.6 & 3.1 & 23.7 & 3.2 & 0.12 & .947 \\
\hline ACE & 16.5 & 2.0 & 16.5 & 3.6 & 17.2 & 3.9 & 17.6 & 1.8 & 0.28 & .836 \\
\hline BAS & 7.6 & 2.5 & 7.7 & 2.8 & 8.4 & 2.9 & 8.2 & 1.9 & 0.15 & .929 \\
\hline $\operatorname{Sub}(+)$ & 48.6 & 4.0 & 48.0 & 8.9 & 49.2 & 6.4 & 49.6 & 4.1 & 0.10 & .956 \\
\hline APA & 3.3 & 2.9 & 5.0 & 5.0 & 4.2 & 1.6 & 5.8 & 4.1 & 0.56 & .644 \\
\hline PRP & 8.2 & 4.0 & 10.5 & 4.1 & 7.8 & 4.6 & 8.6 & 2.9 & 0.85 & .475 \\
\hline HD & 6.8 & 3.0 & 9.2 & 4.0 & 9.2 & 3.8 & 10.0 & 1.8 & 1.31 & .289 \\
\hline $\mathrm{N}$ & 3.0 & 2.0 & 4.8 & 2.8 & 3.4 & 1.9 & 3.8 & 2.7 & 0.93 & .437 \\
\hline Sub(-) & 21.5 & 6.9 & 29.5 & 9.9 & 24.6 & 7.5 & 28.3 & 7.9 & 1.65 & .198 \\
\hline
\end{tabular}

Nota: $n=4$ casos no reportaron el tiempo de diagnóstico. ELA= enfrentamiento y lucha activa, ACE= autocontrol y control emocional, BAS= búsqueda de apoyo social, $\mathrm{Sub}(+)=$ subtotal de las escalas de estrategias de afrontamiento positivas, $\mathrm{APA}=$ ansiedad y preocupación ansiosa, $\mathrm{PRP}=$ pasividad y resignación pasiva, $\mathrm{HD}=$ huida y distanciamiento, $\mathrm{N}=$ negación, $\mathrm{Sub}(-)=$ subtotal de las escalas de estrategias de afrontamiento negativas, BIS= Body Image Scale (Escala de imagen corporal).

Tabla 4. Anova de Imagen corporal y estrategias de afrontamiento según el estadio del cáncer

\begin{tabular}{|c|c|c|c|c|c|c|c|c|c|c|c|c|}
\hline \multirow{3}{*}{ Variable } & \multicolumn{10}{|c|}{ ESTADIO } & \multirow{3}{*}{$\begin{array}{c}F \\
(\mathrm{gl} .4)\end{array}$} & \multirow{3}{*}{$p$} \\
\hline & \multicolumn{2}{|c|}{$\begin{array}{c}0 / \text { in situ } \\
(n=5)\end{array}$} & \multicolumn{2}{|c|}{$\begin{array}{c}\mathrm{I} \\
(n=10)\end{array}$} & \multicolumn{2}{|c|}{$\begin{array}{c}\mathrm{II} \\
(n=10)\end{array}$} & \multicolumn{2}{|c|}{$\begin{array}{c}\text { III } \\
(n=10)\end{array}$} & \multicolumn{2}{|c|}{$\begin{array}{c}\mathrm{IV} \\
(n=2)\end{array}$} & & \\
\hline & $M$ & $D E$ & $M$ & $D E$ & $M$ & $D E$ & $M$ & $D E$ & $M$ & $D E$ & & \\
\hline \multicolumn{13}{|c|}{ Imagen corporal } \\
\hline BIS & 21.4 & 6.7 & 15.5 & 2.7 & 20.5 & 8.9 & 18.2 & 4.9 & 23.0 & 18.3 & 1.10 & .373 \\
\hline \multicolumn{13}{|c|}{ estrategIas de afrontamIento } \\
\hline ELA & 24.8 & 1.4 & 23.9 & 3.1 & 22.6 & 3.7 & 23.1 & 4.3 & 26.5 & 0.7 & 0.75 & .561 \\
\hline ACE & 18.8 & 1.7 & 17.3 & 2.2 & 15.7 & 3.6 & 16.0 & 3.7 & 18.0 & 4.2 & 1.10 & .371 \\
\hline BAS & 7.6 & 3.0 & 7.8 & 2.2 & 8.5 & 2.7 & 7.3 & 3.2 & 9.5 & 3.5 & 0.39 & .810 \\
\hline $\operatorname{Sub}(+)$ & 51.2 & 5.4 & 49.0 & 4.4 & 46.8 & 8.7 & 46.4 & 9.5 & 54.0 & 8.4 & 0.72 & .580 \\
\hline APA & 4.8 & 4.8 & 3.1 & 3.1 & 7.0 & 4.9 & 5.8 & 3.1 & 0.5 & 0.7 & 1.99 & .119 \\
\hline PRP & 11.4 & 2.8 & 8.2 & 3.1 & 8.9 & 4.5 & 7.5 & 3.2 & 13.5 & 2.1 & 1.94 & .126 \\
\hline HD & 9.8 & 3.4 & 7.5 & 2.3 & 9.5 & 4.6 & 9.7 & 2.1 & 7.0 & 4.2 & 0.92 & .460 \\
\hline $\mathrm{N}$ & 4.6 & 2.9 & 3.5 & 2.7 & 4.0 & 2.4 & 2.9 & 1.7 & 7.0 & 1.4 & 1.41 & .250 \\
\hline $\operatorname{Sub}(-)$ & 30.6 & 3.7 & 22.3 & 6.0 & 29.4 & 12.7 & 25.90 & 7.4 & 28.0 & 0.0 & 1.19 & .332 \\
\hline
\end{tabular}

Nota: $\mathrm{ELA}=$ enfrentamiento y lucha activa, $\mathrm{ACE}=$ autocontrol y control emocional, BAS= búsqueda de apoyo social, Sub(+)= subtotal de las escalas de estrategias de afrontamiento positivas, $\mathrm{APA}=$ ansiedad y preocupación ansiosa, $\mathrm{PRP}=$ pasividad y resignación pasiva, $\mathrm{HD}=$ huida y distanciamiento, $\mathrm{N}=$ negación, $\operatorname{Sub}(-)=$ subtotal de las escalas de estrategias de afrontamiento negativas, BIS= Body Image Scale (Escala de imagen corporal). 
Tabla 5. Relación entre afrontamiento (positivas y negativas) e imagen corporal

\begin{tabular}{|c|c|c|c|c|c|c|c|c|c|c|}
\hline & \multicolumn{3}{|c|}{ Estrategias positivas } & \multicolumn{4}{|c|}{ Estrategias negativas } & \multicolumn{2}{|c|}{ Subtotales } & \multirow{2}{*}{$\begin{array}{c}\text { IC } \\
\text { BIS }\end{array}$} \\
\hline & ELA & $\mathrm{ACE}$ & BAS & APA & PRP & HD & $\mathrm{N}$ & $\operatorname{Sub}(+)$ & $\operatorname{Sub}(-)$ & \\
\hline ELA & 1 & $.73 * *$ & .25 & $-.39 *$ & .16 & -.00 & $.41 * *$ & $.86 * *$ & -.00 & -.10 \\
\hline ACE & & 1 & $.35^{*}$ & -.25 & .10 & .26 & .28 & $.88 * *$ & .10 & -.29 \\
\hline BAS & & & 1 & .09 & .11 & .11 & -.04 & $.63^{* *}$ & .12 & -.28 \\
\hline APA & & & & 1 & .18 & $.64 * *$ & $-.34 *$ & -.25 & $.70 * *$ & .11 \\
\hline PRP & & & & & 1 & .29 & $.35^{*}$ & .16 & $.72 * *$ & .04 \\
\hline HD & & & & & & 1 & -.16 & .15 & $.76^{* *}$ & -.10 \\
\hline $\mathrm{N}$ & & & & & & & 1 & .29 & .21 & -.00 \\
\hline $\operatorname{Sub}(+)$ & & & & & & & & 1 & .09 & -.27 \\
\hline $\operatorname{Sub}(-)$ & & & & & & & & & 1 & .03 \\
\hline BIS & & & & & & & & & & 1 \\
\hline
\end{tabular}

Nota: $\mathrm{ELA}=$ enfrentamiento y lucha activa, $\mathrm{ACE}=$ autocontrol y control emocional, BAS $=$ búsqueda de apoyo social, Sub $(+)=$ subtotal estrategias de afrontamiento positivas, $\mathrm{APA}=$ ansiedad y preocupación ansiosa, $\mathrm{PRP}=$ pasividad y resignación pasiva, $\mathrm{HD}=$ huida y distanciamiento, $\mathrm{N}=$ negación, $\mathrm{Sub}(-)=$ subtotal estrategias de afrontamiento negativas, IC = Imagen corporal, BIS= Body Image Scale (Escala de imagen corporal).* $\mathrm{p}<.05, * * \mathrm{p}<.01$.

\section{Relación entre la imagen corporal y las estrategias de afrontamiento}

Los coeficientes de correlación de los puntajes entre la IC y las EA no se relacionaron. No obstante, cabe destacar que las escalas del CAEPO se relacionaron entre sí: El subtotal de EA positivas se relacionó con las subescalas ELA $(r=.86$, $p<.01)$, ACE $(r=.88, p<.01)$ y BAS $(r=.63, p<.01)$. Igualmente, el subtotal de EA negativas se relacionó con tres escalas: APA $(r=.70, p<.01), \mathrm{PRP}(r=.72, p<.01) \mathrm{y}$ $\mathrm{HD}(r=.76, p<.01)$.

\section{Discusión}

Este estudio tuvo los propósitos de estudiar las posibles diferencias en la IC y las EA en función a variables médico quirúrgicas (intervención quirúrgica, tiempo de diagnóstico y estadio) y analizar la posible relación entre la IC y las EA en mujeres con cáncer de seno.

Frente al primer objetivo, los resultados revelan que la IC y las EA no son cambiantes en función a la intervención quirúrgica, el tiempo de diagnóstico y estadio oncológico. $\mathrm{Al}$ respecto, los estudios previos han resaltado la asociación entre el tipo de cirugía y la IC, señalando que la mastectomía genera mayor deterioro de ésta (Olivares et al., 2010) y presenta mayor afectación cognitiva, afectiva y conductual que la cirugía conservadora (Fobair et al., 2006; Olivares et al., 2010; Shoma et al., 2009); mientras que la reconstrucción mamaria contribuye a restaurarla (Den Heijer et al., 2012; Marin \& Sanchez, 2010; Rincón et al., 2012). Por el contrario, aunque determinadas áreas de la IC (actitudes frente a la propia desnudez y el atractivo físico) sí se ven afectadas, la mastectomía por cáncer de mama llevada a cabo de forma global no se asocia con una peor autovaloración en dimensiones como el estado de salud, la apariencia física y la capacidad funcional (Segura-Valverde, García-Nieto, \& Saúl, 2014)

Nuestros resultados no apoyan dichas tesis y parecen ser paradójicos, pues las pacientes que no han recibido ninguna intervención quirúrgica evidencian mayor detrimento de su IC que aquellas que sí la han recibido. Lo anterior puede estar en dirección al modelo del sentido común de Leventhal $(39,40)$ donde configuran esquemas cognitivos de creencias en el binomio salud-enfermedad, por ejemplo, otras variables como por ejemplo apoyo socioemocional de la pareja configuran problemas cognitivos-conductuales y dificultades de adaptación. Además, es probable que el aspecto más relevante en dicho proceso no sea el cambio quirúrgico en sí mismo, sino su significado en cuanto a la representación psicológica del cuerpo, la normalidad, feminidad y sensualidad. De hecho, se ha reportado que los cambios en la IC no son notorios en los primeros tres años posquirúrgicos (Fallbjörk, Rasmussen, Karlsson \& Salander, 2012). Igualmente, algunas variables sociodemográficas, como la edad, la educación y el estado marital 
han mostrado ser predictores de la calidad de vida de los pacientes y sus familiares (King, Kenny, Shiell, Hall \& Boyages, 2000).

Adicionalmente, los antecedentes investigativos sobre la relación entre la IC y el tiempo de diagnóstico parecen ser contrapuestos. Algunos han reportado que a mayor tiempo de diagnóstico, mejor IC (Rincón et al., 2012); mientras otros evidencian que las mujeres con mayor tiempo de diagnóstico tienen una IC más pobre que aquellas con diagnóstico menor a un año (Hartl et al., 2003). Nuestros hallazgos no confluyen con los estudios previos, mostrando que la IC es estable en función al tiempo de dictamen oncológico. Asimismo, observamos homogeneidad de la IC según el estadio del cáncer de seno. Lo cual difiere con el único estudio encontrado sobre el tema, que reporta que a mayor estadio oncológico, mayor deterioro de la IC (Hartl et al., 2003). Sin embargo, cabe acotar que dicho estudio únicamente añadió dos ítems de IC a un cuestionario de calidad de vida, por lo que se cuestiona la validez de sus conclusiones.

Respecto a las EA, algunos estudios han analizado su relación con el tipo de tratamiento (De-Haro et al., 2014; Zucca et al., 2010) encontrando que la intervención quirúrgica implica la sobreestimación de las amenazas (Tesson et al., 2016), aunque se tiene en cuenta la aparición de factores de estrés en diversas etapas posquirúrgicas (Jørgensen, Garne, Søgaard \& Laursen, 2015; Jørgensen, Laursen, Garne, Sherman \& Søgaard, 2016). Similarmente, no se encontraron estudios sobre las EA según el estadio oncológico y el tratamiento, aunque sí relacionados con la percepción de supervivencia y el riesgo de muerte en ausencia versus intervención quirúrgica mostrando mejor manejo de la situación en aquellas con presencia de mutación mamaria bilateral. También se ha mostrado que las decisiones para el manejo de la situación no tienen clara relación con el tipo de tratamiento quirúrgico, sino con el nivel de riesgo de la enfermedad (Baker, Mayer \& Esposito, 2013). Por lo tanto, esta investigación extiende la literatura, concluyendo que las EA no varían según el tipo de cirugía, ni el estadio de la enfermedad. Finalmente, nuestros resultados evidencian uniformidad en las EA en función al tiempo de diagnóstico oncológico, lo cual diside de lo informado por otros autores (Geyer, Koch-giesselmann \& Noeres, 2015), que han mostrado que el afrontamiento en respuesta a éste varía notablemente en el tiempo; en tantos otros (Nosarti et al., 2002) sostienen que el uso de EA decrece con el paso del tiempo; o que el uso de algunas EA decrece, mientras el de otras incrementa (Danhauer et al., 2009).

De acuerdo a todo lo anterior, la inconsistencia entre los reportes de la literatura y nuestros resultados pueden explicarse por dos aspectos. Primero, por los factores personales asociados a la historia de vida de cada paciente, tales como resiliencia, autoeficacia, espiritualidad entre otros, que amortiguan el impacto psicológico de situaciones traumáticas o altamente estresantes. Por lo tanto, la variabilidad de la IC y la forma en que se afronta parecen relacionarse con características individuales y personales, descartando de momento el impacto de variables médico quirúrgicas, tales como la intervención quirúrgica, tiempo de diagnóstico y estadio. Segundo, por aspectos metodológicos, particularmente el uso de diferentes instrumentos de medida. Por esto, resulta interesante revisar las evidencias psicométricas de especificidad de tales herramientas en condiciones particulares en futuros estudios.

Frente al segundo objetivo, los resultados no muestran una relación entre la IC y las EA. En esta línea, aunque la literatura es escasa, estudios previos han reportado que sí bien no hay asociación entre dichas variables, las pacientes con mejor IC, sí presentan mayor autoeficacia (habilidad percibida para afrontar; Pikler \& Winterowd, 2003). Cabe destacar que si bien nuestros resultados no muestran significancia estadística, que el grupo de escalas de EA positivas (enfrentamiento y lucha activa, autocontrol y control emocional, búsqueda de apoyo social) tiene un peso relacional inverso al deterioro de la IC, lo cual contrasta en que mujeres con mayor desesperanza, relaciones familiares débiles y bajo autoconcepto requieren mayor apoyo social, aunque el nivel de satisfacción con el mismo es más crítico que el tamaño de la red de apoyo (Chen \& Chang, 2012).

En resumen, resaltamos que el aporte de nuestros datos alimenta el cuerpo del conocimiento de la Psicología de la Salud, específicamente a la Psico-oncología, ya que apoya a los profesionales de estos campos en la comprensión las EA y la IC y el desarrollo de intervenciones eficaces e integrales tanto con el paciente, como con su familia durante la evolución de la enfermedad. Adicionalmente, destacamos el tamaño muestral como principal limitación de este trabajo, por lo que subrayamos que los resultados deben asumirse con prudencia. Como se señaló anteriormente, ésta es la primera fase de una línea de investigación macro que pretende recolectar muestras en otras ciudades de Colombia; por lo tanto, nuestra proyección es ampliar el tamaño de la muestra, con el fin de lograr mayor poder estadístico, alcance metodológico y extensión de los hallazgos, además de hacer extenso a otro tipos de cáncer. Nuestros resultados nos permiten concluir que la IC y las EA no presentan diferencias según de intervención quirúrgica, el tiempo de diagnóstico y estadio oncológico. No se halló relación la IC y la EA. 


\section{Referencias}

Aguirre-Loaiza, H. H., Núñez, C., Navarro, A. M., \& Cortés, S. (2017). Calidad de vida según el estadio del cáncer de seno en mujeres: Análisis desde el FACT-B y SF-36. Psychologia, 11(1), 109-120

Ahmed, K., Marchand, E., Williams, V., Coscarelli, A., \& Ganz, P. A. (2016). Development and pilot testing of a psychosocial intervention program for young breast cancer survivors. Patient Education and Counseling, 99, 414-420. https://doi.org/10.1016/j.pec.2015.09.014

Baker, S. K., Mayer, D. K., \& Esposito, N. (2013). The Contralateral Prophylactic Mastectomy Decision-Making Process. Plastic Surgical Nursing, 33, 11-21. https://doi.org/10.1097/PSN.0b013e3182842424

Barnaś, E., Skręt-Magierło, J., Skręt, A., \& Bidziński, M. (2012). The quality of life of women treated for cervical cancer. European Journal of Oncology Nursing: The Official Journal of European Oncology Nursing Society, 16, 59-63. https://doi.org/10.1016/j.ejon.2011.03.001 Brown, L. F., \& Kroenke, K. (2009). Cancer-related fatigue and its associations with depression and anxiety: a systematic review. Psychosomatics, 50(5),440-7. https://doi.org/10.1176/appi.psy.50.5.440

Brunet, J., Sabiston, C. M., \& Burke, S. (2013). Surviving breast cancer: Women's experiences with their changed bodies. Body Image, 10, 344-351. https://doi.org/10.1016/j.bodyim.2013.02.002

Burgess, C., Cornelius, V., Love, S., Graham, J., Richards, M., \& Ramirez, A. (2005). Depression and anxiety in women with early breast cancer: five year observational cohort study. British Medical Journal, 330(7493), 702. https://doi.org/10.1136/bmj.38343.670868.D3

Chen, P. Y., \& Chang, H.-C. (2012). The coping process of patients with cancer. European Journal of Oncology Nursing: The Official Journal of European Oncology Nursing Society, 16, 10-6. https:// doi.org/10.1016/j.ejon.2011.01.002

Colegio Colombiano de Psicólogos (2012). Deontología y Bioética del ejercicio de la Psicología en Colombia. Bogotá DC.

Danhauer, S. C., Crawford, S. L., Farmer, D. F., \& Avis, N. E. (2009). A longitudinal investigation of coping strategies and quality of life among younger women with breast cancer. Journal of Behavioral Medicine, 32, 371-379. https://doi.org/10.1007/s10865-009-9211-x

De-Haro, M. A., Gallardo-Vidal, L., Martínez-Martínez, M. L., Camacho-Calderon, N., Velazquez-Tlapanco, J., \& Paredes, E. (2014). Factores relacionados con las diferentes estrategias de afrontamiento al cáncer de mama en pacientes de reciente diagnóstico. Psicooncología, 11, 87-99. https://doi.org/10.5209/rev_PSIC.2014.v11.n1.44919

Den Heijer, M., Seynaeve, C., Timman, R., Duivenvoorden, H. J., Vanheusden, K., Tilanus-Linthorst, M., ... Tibben, A. (2012). Body image and psychological distress after prophylactic mastectomy and breast reconstruction in genetically predisposed women: A prospective longterm follow-up study. European Journal of Cancer, 48, 1263-1268. https://doi.org/10.1016/j.ejca.2011.10.020

Fallbjörk, U., Rasmussen, B. H., Karlsson, S., \& Salander, P. (2012). Aspects of body image after mastectomy due to breast cancer - A two-year follow-up study. European Journal of Oncology Nursing, 17, 340-345. https://doi.org/10.1016/j.ejon.2012.09.002

Fobair, P., Stewart, S. L., Chang, S., D’Onofrio, C., Banks, P. J., \& Bloom, J. R. (2006). Body image and sexual problems in young women with breast cancer. Psycho-Oncology, 15, 579-594. https:// doi.org/10.1002/pon.991
Folkman, S., Lazarus, R. S., Gruen, R. J., \& Delongis, A. (1986). Appraisal, Coping, Health Status, and Psychological Symptoms, 50, 571-579.

Font, A., \& Cardoso, A. (2009). Afrontamiento en cáncer de mama: pensamientos, conductas y reacciones emocionales. Psicooncologia, $6,27-42$.

Geyer, S., Koch-Giesselmann, H., \& Noeres, D. (2015). Coping with breast cancer and relapse: Stability of coping and long-term outcomes in an observational study over 10 years. Social Science y Medicine, 135, 92-98. https://doi.org/10.1016/j.socscimed.2015.04.027

González, M. T. (2004). Cuestionario de afrontamiento al estrés para pacientes oncológicos -CAEPO. Madrid: Ediciones TEA.

González, M. T. (2008a). Análisis de un instrumento de evaluación del afrontamiento del estrés en sujetos con cáncer Analysis of an instrument to assess coping with stress by subjects suffering cancer. Revista Iberoamericana de Diagnóstico Y Evaluación Psicológica, 1, 35-49.

González, M. T. (2008b). Análisis de un instrumento de evaluación del afrontamiento del estrés en sujetos con cáncer Analysis of an instrument to assess coping with stress by subjects suffering cancer. Revista Iberoamericana de Diagnóstico Y Evaluación Psicológica, 1,35-49.

Hartl, K., Janni, W., Kastner, R., Sommer, H., Strobl, B., Rack, B., \& Stauber, M. (2003). Impact of medical and demographic factors on longterm quality of life and body image of breast cancer patients. Annals of Oncology, 14, 1064-1071. https://doi.org/10.1093/annonc/mdg289

Hopwood, P., Fletcher, I., Lee, A., \& Al Ghazal, S. (2001). A body image scale for use with cancer patients. European Journal of Cancer (Oxford, England: 1990), 37, 189-97. https://doi.org/10.1016/S09598049(00)00353-1

Hopwood, P., Haviland, J., Mills, J., Sumo, G., \& Bliss, J. (2007). The impact of age and clinical factors on quality of life in early breast cancer: An analysis of 2208 women recruited to the UK START Trial (Standardisation of Breast Radiotherapy Trial). Breast, 16(3), 241-251. https://doi.org/10.1016/j.breast.2006.11.003

Jørgensen, L., Garne, J. P., Søgaard, M., \& Laursen, B. S. (2015). The experience of distress in relation to surgical treatment and care for breast cancer: An interview study. European Journal of Oncology Nursing, 19, 612-618. https://doi.org/10.1016/j.ejon.2015.03.009

Jørgensen, L., Laursen, B. S., Garne, J. P., Sherman, K. A., \& Søgaard, M. (2016). Prevalence and predictors of distress in women taking part in surgical continuity of care for breast cancer: A cohort study. European Journal of Oncology Nursing, 22, 30-36. https://doi.org/10.1016/j. ejon.2016.01.004

Juárez, D., \& Landero, R. (2011). Imagen corporal, funcionamiento sexual, autoestima y optimismo en mujeres con cáncer de mama. Nova Scientia, 4, 16-34. Retrieved from https://dialnet.unirioja.es/servlet/ articulo? codigo $=3785174$ yinfo $=$ resumenyidioma $=$ SPA

King, M. T., Kenny, P., Shiell, A., Hall, J., \& Boyages, J. (2000). Quality of Life Three Months and One Year after First Treatment for Early Stage Breast Cancer: Influence of Treatment and Patient Characteristics. Quality of Life Research, 9, 789-800.

Marin, M., \& Sánchez, A. (2010). Reconstructive surgery in young women with breast cancer. Breast Cancer Research and Treatment, 123, 67-74. https://doi.org/10.1007/s10549-010-1127-1 
Mcdonough, M. H., Sabiston, C. M., \& Wrosch, C. (2014). Predicting changes in posttraumatic growth and subjective well-being among breast cancer survivors: The role of social support and stress. Psycho-Oncology, 23, 114-120. https://doi.org/10.1002/pon.3380

Mera, P.C., \& Ortiz, M. (2012). La relación del optimismo y las estrategias de afrontamiento con la calidad de vida de mujeres con cáncer de mama. [The relationship of optimism and coping strategies with the quality of life of women with breast cancer.]. Terapia Psicológica, 30, 69-78. https://doi.org/10.4067/S0718-48082012000300007

Ministerio de Salud - República de Colombia. (1993). Resolución 8430, 1993 Ministerio De Salud Resolucion 1-19. Bogotá DC.

Ministerio de Salud y Protección Social. (2014). Cáncer de mama, una enfermedad en ascenso en Colombia. Retrieved from https://www. minsalud.gov.co/Paginas/-Cancer-de-mama,-una-enfermedad-enascenso-en-Colombia.aspx

Moreira, H., \& Canavarro, M. C. (2010). A longitudinal study about the body image and psychosocial adjustment of breast cancer patients during the course of the disease. European Journal of Oncology Nursing, 14, 263-270. https://doi.org/10.1016/j.ejon.2010.04.001

Moreira, H., Silva, S., Marques, A., \& Canavarro, M. C. (2010). The Portuguese version of the Body Image Scale (BIS) - psychometric properties in a sample of breast cancer patients. European Journal of Oncology Nursing, 14, 111-118. https://doi.org/10.1016/j.ejon.2009.09.007

Nosarti, C., Roberts, J. V, Crayford, T., McKenzie, K., \& David, A. S. (2002). Early psychological adjustment in breast cancer patients. Journal of Psychosomatic Research, 53(6), 1123-1130. https://doi. org/10.1016/S0022-3999(02)00350-1

Olivares, M., Martin, M. D., Roman, J. M., \& Moreno, A. (2010). Valoración biopsicosocial en pacientes con patología mamaria oncológica quirurgica. Psicooncologia, 7, 81-97.

Pikler, V., \& Winterowd, C. (2003). Racial and body image differences in coping for women diagnosed with breast cancer. Health Psychology: Official Journal of the Division of Health Psychology, American Psychological Association, 22, 632-7. https://doi.org/10.1037/02786133.22.6.632

Rincón, M. E., Pérez, M. A., Borda, M., \& Martín, A. (2012). Impacto de la reconstrucción mamaria sobre la autoestima y la imagen corporal en pacientes con cáncer de mama. Universitas Psychologica, 11, 25-42. Retrieved from http://revistas.javeriana.edu.co/index.php/ revPsycho/article/view/575

Segura-Valverde, M., García-Nieto, R., \& Saúl, L. A. (2014). Imagen Corporal y autoestima en mujeres mastectomizadas. Psicooncologia, 11, 45-57. https://doi.org/10.5209/rev

Shoma, A. M., Mohamed, M. H., Nouman, N., Amin, M., Ibrahim, I. M., Tobar, S. S., ... William, S. G. (2009). Body image disturbance and surgical decision making in egyptian post menopausal breast cancer patients. World Journal of Surgical Oncology, 7, 66. https:// doi.org/10.1186/1477-7819-7-66

Silva, S. M., Crespo, C. \& Canavarro, M. C. (2012). Pathways for psychological adjustment in breast cancer: A longitudinal study on coping strategies and posttraumatic growth. Psychology y Health, 27(11), 1323-1341. https://doi.org/10.1080/08870446.2012.676644
Tesson, S., Richards, I., Porter, D., Phillips, K. A. K.-A., Rankin, N., Musiello, T., ... Butow, P. (2016). Women's preferences for contralateral prophylactic mastectomy: An investigation using protection motivation theory. Patient Education and Counseling, 99, 814-822. https://doi.org/10.1016/j.pec.2015.11.012

Weinberg, R. (2013). The biology of cancer. New York: Garland Science. White, C. A. (2000). Body image dimensions and cancer: A heuristic cognitive behavioural model. Psycho-Oncology, 9, 183-192. https://doi. org/10.1002/1099-1611(200005/06)9:3<183::AID-PON446>3.0.CO;2-L

WHO. (2015). Cáncer. Nota descriptiva $N^{\circ}$ 297,. Ginebra. Retrieved from http://www.who.int/mediacentre/factsheets/fs297/en/

Zucca, A. C., Boyes, A. W., Lecathelinais, C., \& Girgis, A. (2010). Life is precious and I'm making the best of it: Coping strategies of long-term cancer survivors. Psycho-Oncology, 19(12), 1268-1276. https://doi. org/10.1002/pon. 1686 